\title{
Stabilisation of emulsified Agarwood oil in an aqueous system using non-ionic surfactant
}

\begin{abstract}
Owing to the annually increasing market value of pure agarwood oil, the extractedagarwood oil from Aquilaria malaccensis was emulsified in an aqueous solution using non-ionic surfactant (Tween 80). The surfactant concentration of $0.0167 \%$ was determined as the criticalmicelle concentration (CMC) with an interfacial tension value of $0.014 \mathrm{mNm}-1$. The adsorption of surfactant at the oil/water interface at the CMC value, however, reduced the zeta potential of the emulsified oil from -45 to $-43 \mathrm{mV}$, and increased its size from 85 to $89 \mathrm{~nm}$. Outside of the CMC value, the emulsified oil droplets tended to coalesce, owing to insufficient coverage of the surfactant at oil/water interface and Ostwald ripening. The droplet size distribution and zeta potential value of the emulsified oil droplets produced at the CMC were the most stable over a month of storage. No significant changes in the emulsified droplet size occurred when the $\mathrm{pH}$ conditions varied from $\mathrm{pH} 3$ to 10 . The emulsified droplets images obtained from transmission electron microscopy analysis showed a reduction in the layer thickness of the surfactant from 30 to $10 \mathrm{~nm}$ in acidic condition and 30 to $19 \mathrm{~nm}$ in alkaline condition. The agarwood oil emulsification at CMC value enhance the stability of chemically unstable compounds from degradation.
\end{abstract}

Keyword : Agarwood oil; Nanoemulsion; Critical micelle concentration (CMC); Stability; Surfactant layer thickness. 